\title{
How to Reduce Head CT Orders in Children with Hydrocephalus Using the Lean Six Sigma Methodology: Experience at a Major Quaternary Care Academic Children's Center
}

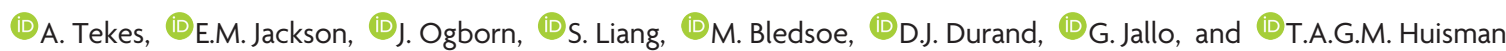

\begin{abstract}
BACKGROUND AND PURPOSE: Lean Six Sigma methodology is increasingly used to drive improvement in patient safety, quality of care, and cost-effectiveness throughout the US health care delivery system. To demonstrate our value as specialists, radiologists can combine lean methodologies along with imaging expertise to optimize imaging elements-of-care pathways. In this article, we describe a Lean Six Sigma project with the goal of reducing the relative use of pediatric head CTs in our population of patients with hydrocephalus by $50 \%$ within 6 months.
\end{abstract}

MATERIALS AND METHODS: We applied a Lean Six Sigma methodology using a multidisciplinary team at a quaternary care academic children's center. The existing baseline imaging practice for hydrocephalus was outlined in a Kaizen session, and potential interventions were discussed. An improved radiation-free workflow with ultrafast MR imaging was created. Baseline data were collected for 3 months by using the departmental radiology information system. Data collection continued postintervention and during the control phase (each for 3 months). The percentage of neuroimaging per technique (head CT, head ultrasound, ultrafast brain MR imaging, and routine brain MR imaging) was recorded during each phase.

RESULTS: The improved workflow resulted in a 75\% relative reduction in the percentage of hydrocephalus imaging performed by $C T$ between the pre- and postintervention/control phases (Z-test, $P=.0001)$.

CONCLUSIONS: Our lean interventions in the pediatric hydrocephalus care pathway resulted in a significant reduction in head CT orders and increased use of ultrafast brain MR imaging.

ABBREVIATIONS: DMAIC = Define, Measure, Analyze, Improve, and Control; LSS = Lean Six Sigma; PED = pediatric emergency department; UF-MRI = ultrafast brain MRI; US = ultrasonography

ean and Six Sigma are 2 prominent quality-improvement -methodologies that have been successfully applied in health care since 1998, with increasing use during the past decade related to the increased emphasis on patient safety and cost-effectiveness in medicine. "1,2 "Six Sigma" is a process initially developed by the Motorola Corporation in 1986, which aims to improve quality by identifying and correcting the causes of errors to reduce the error rate to a six sigma level, (ie, 3.4 defects per million opportunities).

Received September 15, 2015; accepted after revision November 12.

From the Division of Pediatric Radiology and Pediatric Neuroradiology (A.T., D.J.D., T.A.G.M.H.), Department of Radiology (S.L., M.B.), Russell H. Morgan Department of Radiology and Radiological Science; Division of Pediatric Neurosurgery, Department of Neurosurgery (E.M.J., G.J.); and Department of Pediatrics (J.O.), The Johns Hopkins Medical Institutions, Baltimore, Maryland.

Please address correspondence to Aylin Tekes, MD, Division of Pediatric Radiology and Pediatric Neuroradiology, The Russell H. Morgan Department of Radiology and Radiological Science, The Johns Hopkins School of Medicine, Charlotte R.

Bloomberg Children's Center, Sheikh Zayed Tower, Room 4155, 1800 Orleans St, Baltimore, MD 21287-0842; e-mail: atekes1@jhmi.edu

http://dx.doi.org/10.3174/ajnr.A4658
"Lean" is another quality-improvement methodology that evolved from the Toyota Production system in 1990, which uses improvements to eliminate waste and preserve steps that incrementally optimize the "value" created by the process in question. Combined as Lean Six Sigma (LSS), a 5-stage powerful methodology, Define, Measure, Analyze, Improve, and Control (DMAIC), has evolved.

Approximately 39,000 children with hydrocephalus require inpatient admissions annually in the United States. ${ }^{3,4}$ Most or all of these children will require cross-sectional imaging to diagnose and follow their condition. Because CT is currently the mainstay of hydrocephalus imaging at most centers, patients with hydrocephalus have a cumulative radiation exposure much higher than that of the average population. Patients exposed to higher cumulative radiation doses have an increased risk of cancer (especially children), with the brain being the most sensitive to radiation-induced cancers on the basis of recent empiric studies. ${ }^{5-10}$ Disease-specific imaging protocols and pathways to help mitigate unnecessary radiation 
exposure in high-risk/vulnerable populations have been discussed in various medical/scientific forums.

Despite these considerations, radiation-free imaging modalities, such as brain MR imaging and head ultrasonography (US), are often underused due to logistic barriers and/or resource constraints such as lack of a 24/7 in-house MR imaging technologist, the longer examination times of MR imaging compared with CT, MR imaging often requiring sedation/anesthesia in the youngest patients, the higher unit cost of MR imaging, and so forth. Some authors have advocated the use of rapid-sequence MR imaging/ rapid brain MR imaging/quick brain MR imaging (different terminologies have been used; in this article, we will use the term "ultrafast brain MR imaging") in patients with hydrocephalus in the past decade. Ultrafast brain MR imaging (UF-MR imaging) offers reliable visualization of the ventricular catheter and superior anatomic detail of the ventricular system; therefore, diagnostic accuracy of the test is not reduced as the child benefits from radiation-free imaging. ${ }^{11-15}$ However, in our experience, these MR imaging protocols are limited to relatively few centers or are performed only during certain hours of the day.

Because the barriers to using radiation-free imaging more effectively in pediatric hydrocephalus are so frequently logistic and involve complex, multidisciplinary workflows, we took an LSS approach for process improvement. The purpose of this study was to determine whether a multidisciplinary LSS approach could successfully reduce our reliance on head CT in the pediatric hydrocephalus population by $50 \%$ within 6 months, 24/7, from all referring units.

\section{MATERIALS AND METHODS}

In accordance with the Health Insurance Portability and Accountability Act, our institutional review board reviewed the protocol for this prospective study and waived the requirement for informed consent. The Division of Pediatric Radiology and Pediatric Neuroradiology convened a working team with representation from all key stakeholder groups involved in the care of children with hydrocephalus. Our team involved pediatric neuroradiologists, pediatric neurosurgeons, pediatric emergency department (PED) physicians; chief technologists of CT, US, and MR imaging; scheduling staff; a department administrator; and a data analyst. A project leader (a pediatric neuroradiologist) and a physician champion (a pediatric neuroradiologist) were identified. Weekly/bimonthly team meetings were held. We deployed the following 5-step LSS methodology (DMAIC):

1) Define. All team members gathered around the table under the leadership of the project leader and physician champion. After hearing each group member's input, a decision was made on the project title, problem statement, and scope of the work; the project goal was aligned with institutional and departmental priorities (Table 1).

2) Measure. Benchmark data were requested from 2 major national children's hospitals because they had comparable patient profiles, inpatient/outpatient distributions, practitioners, and scanner capabilities compared with our children's center. Both hospitals used a charge modifier to reduce the patient cost for UF-MR imaging by approximately $20 \%$. The benchmark data were used to better understand the perfor-
Table 1: Project charter

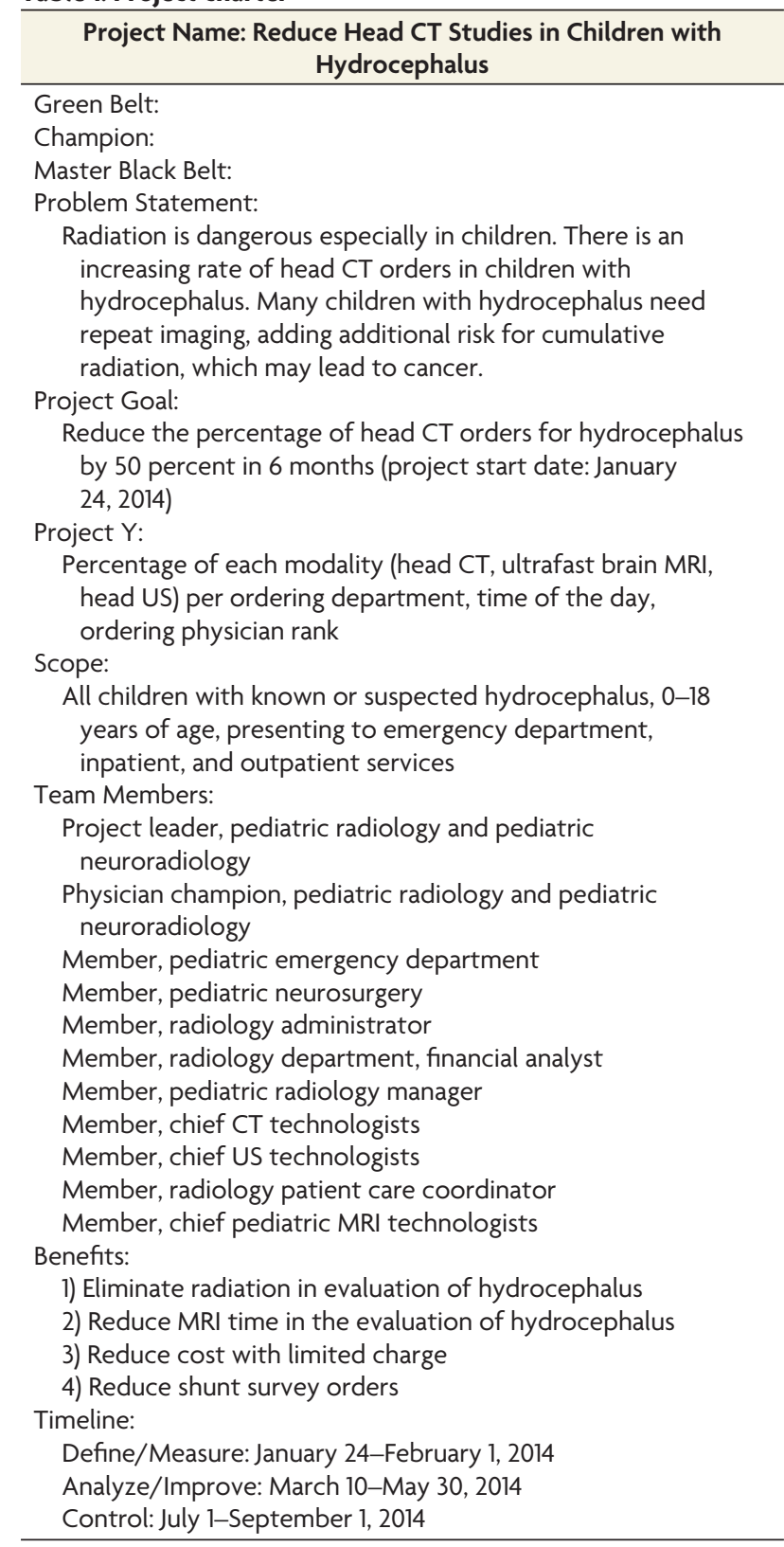

mance with regard to UF-MR imaging. Optimal and practical metrics for defining and measuring the process using institutional/departmental resources were discussed. The departmental radiology information system was searched for the following criteria/key words: hydrocephalus; $0-18$ years of age; and type of imaging performed being head CT, head US, routine brain MR imaging, and UF-MR imaging (with respective examination codes), with date and time of examination and ordering physician rank. Exclusion criteria included acuity of the medical condition requiring an immediate need for transfer to pediatric intensive care unit; the presence of a cochlear implant, lumbar implants/drain, or a Codman device (Codman/Johnson \& Johnson, Raynham, Massachusetts) requiring adjustment after MR imaging; and extreme claustrophobia. Baseline data were collected retrospectively for 3 months on a monthly basis and continued to be collected prospectively 


\section{Patient presents with suspected/known hydrocephalus}

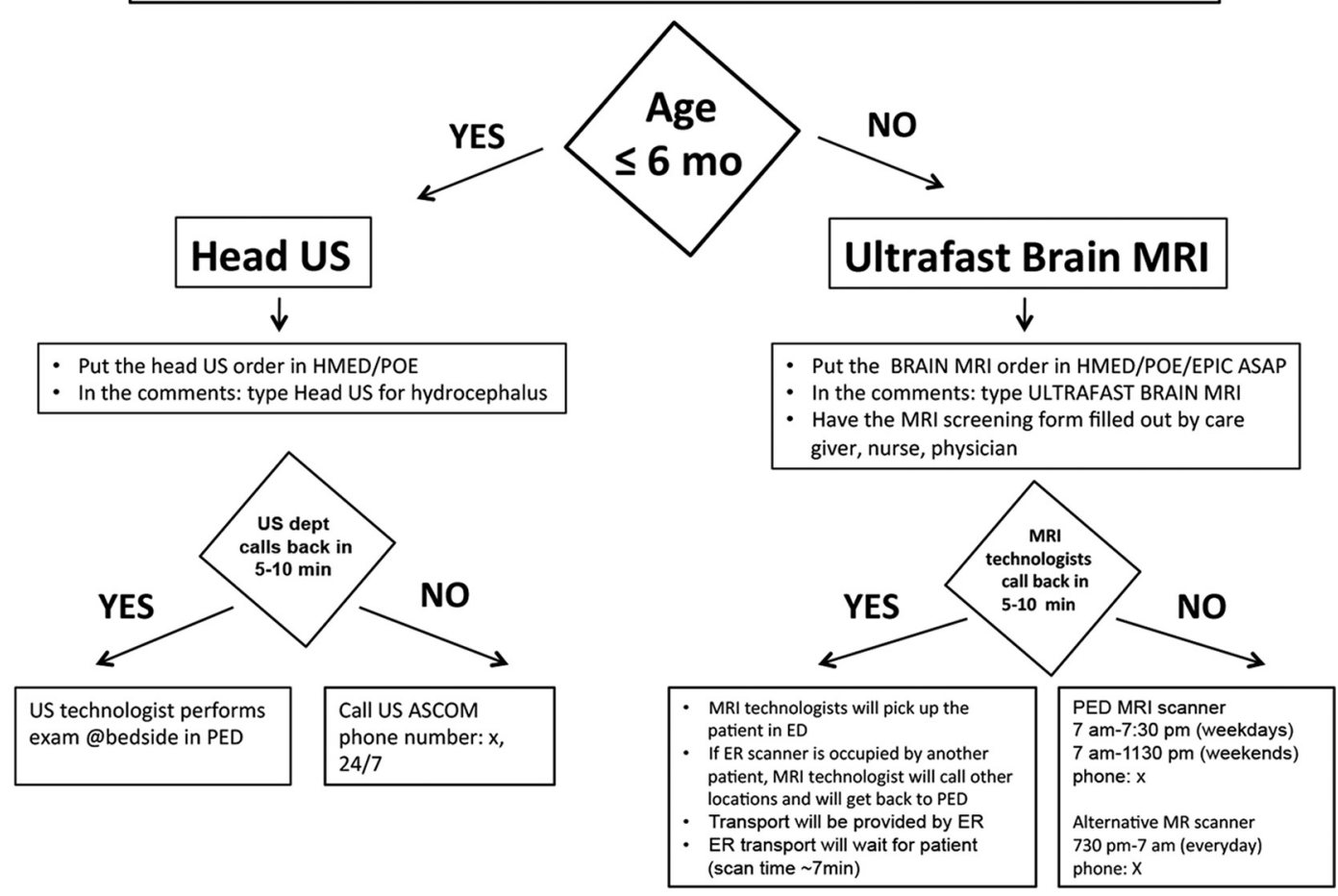

Patients that have programmable shunts: MRI technologist calls on call neurosurgery resident at pediatric neurosurgery pager, $24 / 7$ before they initiate the scan.

FIG 1. Improved workflow for the hydrocephalus imaging pathway. Imaging technologists are required to respond in a timely fashion. In case they could not respond on time, their phone numbers are outlined so that the ordering units can follow-up on their orders. HMED indicates HealthMatics Emergency Department (Allscripts, Chicago, Illinois); POE, Physician Order Entry; EPIC, Epic Systems (Madison, Wisconsin); ASCOM, tel.

during the postintervention/improvement phase and control phase in a similar fashion.

3) Analyze. The percentages of head CT, head US, routine brain MR imaging, and UF-MR imaging examinations were calculated during the baseline, improvement, and control phases. The percentage of the above-mentioned orders was analyzed per ordering unit (PED, inpatient, outpatient), per time of practice (routine day time, after hours, and weekends), and per rank of ordering physician (resident/fellow versus attending), to identify the target areas for improvement.

4) Improve. A visual flow chart for improved hydrocephalus imaging workflow was created (Fig 1) by the team and was implemented during the postintervention phase. Gemba was visited. Four major interventions were identified. Data collection continued for 3 months.

5) Control. Existing interventions were consistently pursued, and data were recorded. The electronic ordering systems were revisited during the control phase.

\section{Lean Interventions}

Lean interventions were categorized as follows: workflow changes and communication, technology improvement, equipment/process improvement, and reducing patient charges for UF-MR imaging.

Workflow Changes and Communication. Education of all units with the new workflow was performed via an in-person visit to the PED, inpatient, and outpatient units during their internal departmental meetings by the project leader. The radiologic value of
UF-MR imaging in pediatric hydrocephalus was explained in detail. Limitations of UF-MR imaging in clinical presentations other than hydrocephalus were explained in detail, such as but not limited to assessment of hemorrhage or ischemia, postoperative tumor assessment, and developmental milestone evaluations. In addition an electronic e-mail alert was sent to all residents, fellows, and attending physicians summarizing the new workflow (Fig 1). The new workflow was printed and placed over each ordering station for easy visual display of information. The electronic ordering system was revisited by the PED physician and pediatric neuroradiologist prioritizing head US and UF-MR imaging in the list of examination choices in the hydrocephalus imaging ordering package with a "pop-up" information box summarizing the improved imaging workflow.

Technology Improvements. Optimization of the existing UF-MR imaging protocol was undertaken to reduce the total MR imaging time to $<5$ minutes while improving image quality using a triplanar T2-weighted HASTE sequence (Fig 2). Due to the short acquisition time, no sedation or anesthesia was used. This process was led by the project leader, physician champion, and the chief MR imaging technologist. The chief MR imaging technologist updated all MR imaging scanners with the optimized protocol and trained/informed all technologists.

Equipment/Process Improvements. A separate shunt programmer was provided to the PED by pediatric neurosurgery. In-service training to the PED staff was given by the manufacturer of the shunt 
programmer. The imaging technologists were required to respond within 5-10 minutes of placement of imaging orders in our radiology information system. MR imaging technologists were required to page the pediatric neurosurgery resident before initiating the MR imaging. All UF-MR images were obtained in the dedicated pediatric MR imaging scanner except when the scanner was occupied by other medical emergencies. In such cases, MR imaging technologists were responsible for identifying alternative available scanners and directing patient care. The process did not require new staff hiring or new job creation. Diagnostic image quality was obtained in all UF-MR imaging studies. No patient required a head CT after undergoing UF-MR imaging (ie, UF-MR images were diagnostically adequate in all patients in this study).

Reduce Patient Charges for UF-MR Imaging. The cost of UF-MR imaging was initially equal to that of routine brain MR imaging. A "charge modifier" was created by the administrator of Billing, Coding, and Compliance to reduce the technical fee.

Statistical significance in the percentage change of head CTs from baseline to improvement and control phases was calculated by using $Z$-test. Attribute capability analysis was performed to predict the long-term effects of the improved results (Minitab Statistical Software; Minitab, State College, Pennsylvania).

\section{RESULTS}

We had 794 patients, of whom 25 were excluded due to following reasons: claustrophobia $(n=6)$, medical emergency to evaluate the ventricular size and shunt positioning in which MR imaging

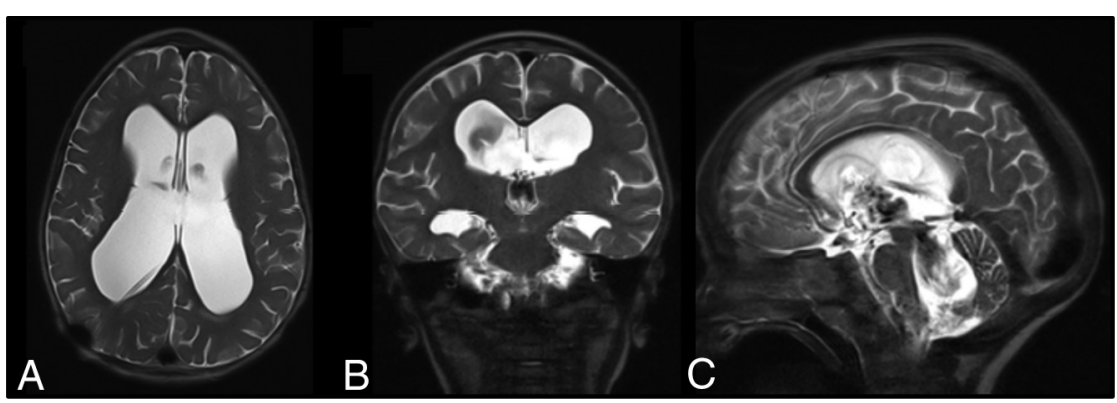

FIG 2. Ultrafast brain MR imaging protocol: axial $(A)$, coronal $(B)$, and sagittal $(C)$ T2-weighted HASTE of the brain. Note the clear visualization of the ventricular system and catheter tip. scanner availability could not meet the medical acuity $(n=4)$, concern for intracranial hemorrhage $(n=10)$, seizures $(n=3)$, and the presence of a lumbar drain for which the provider was uncomfortable with drain settings $(n=2)$. Of the 769 patients, the mean age was $5.3 \pm 6$ years.

Benchmark data were received from 2 major national children's hospitals, where the distribution of head CTs was less and UF-MR imaging was more than our existing baseline data (we are not allowed to share the actual numbers or percentages), indicating the timeliness of improvement in our pediatric hydrocephalus imaging pathway. One hospital reported that most of the UF-MRs were obtained on outpatients, whereas the other hospital reported that UF-MRs were predominantly done on patients in the emergency department.

In our children's center during baseline, the PED was the unit with the highest percentage of head CT orders (86.6\%); $62 \%$ of all baseline orders including all neuroimaging modalities came from residents or fellows (Table 2). Most imaging (67\%) was performed during regular work hours (7 AM to 5 PM) (Table 3); however, when we focused on the PED, most the studies were performed after hours (5:01 PM to 6:59 $\mathrm{AM}$ ) and on weekends (Saturday and Sunday).

There was a statistically highly significant $75 \%$ relative decrease in the total head CT examinations from baseline to the control phase, surpassing our goal of $50 \%$ reduction $(Z$-test, $P<$ .0001 ) (Table 4 ). The goal was achieved at 3 months from deployment of the project. UF-MR imaging examinations increased from $18 \%$ during baseline to $50 \%$ during improvement and control phases, showing a nearly 3 -fold increase. Although there was a minimal increase in head CT and a minimal decrease in UF-MR imaging orders during the first 2 months of the control phase, similar percentages to the improvement phase were reached at the third month of the control phase.

There was a decreasing trend in head US studies, with favored use of UF-MR imaging. The weekly changes in head CT orders from baseline to control phases are summarized in a p-chart in Fig 3. Attribute capability analysis showed that the head CT examination reduction at the final control phase almost reached the 95\%

Table 2: Number and percentage of each modality per rank of ordering physician from baseline to control phases ${ }^{\mathrm{a}}$

\begin{tabular}{|c|c|c|c|c|c|c|c|c|c|c|c|c|c|c|}
\hline & \multicolumn{2}{|c|}{ Baseline } & \multicolumn{2}{|c|}{ I-Phase I } & \multicolumn{2}{|c|}{ I-Phase II } & \multicolumn{2}{|c|}{ I-Phase III } & \multicolumn{2}{|c|}{ C-Phase I } & \multicolumn{2}{|c|}{ C-Phase II } & \multicolumn{2}{|c|}{ C-Phase III } \\
\hline & No. & $\%$ & No. & $\%$ & No. & $\%$ & No. & $\%$ & No. & $\%$ & No. & $\%$ & No. & $\%$ \\
\hline \multicolumn{15}{|l|}{ Attending } \\
\hline RB-MRI & 16 & 20.3 & 4 & 26.7 & 5 & 18.5 & 3 & 10.0 & 14 & 20.9 & 6 & 8.8 & 9 & 12.7 \\
\hline UF-MRI & 18 & 22.8 & 4 & 26.7 & 16 & 59.3 & 10 & 33.3 & 31 & 46.3 & 34 & 50.0 & 38 & 53.5 \\
\hline Head CT & 16 & 20.3 & 2 & 13.3 & & 0.0 & 1 & 3.3 & 4 & 6.0 & 6 & 8.8 & 3 & 4.2 \\
\hline Head US & 29 & 36.7 & 5 & 33.3 & 6 & 22.2 & 16 & 53.3 & 18 & 26.9 & 22 & 32.4 & 21 & 29.6 \\
\hline Attending total & 79 & 38.5 & 15 & 24.2 & 27 & 31.4 & 30 & 32.6 & 67 & 70.5 & 68 & 58.1 & 71 & 63.4 \\
\hline \multicolumn{15}{|l|}{ Resident } \\
\hline RB-MRI & 22 & 17.5 & 5 & 10.6 & 5 & 8.5 & 5 & 8.1 & 4 & 14.3 & 5 & 10.2 & 7 & 17.1 \\
\hline UF-MRI & 19 & 15.1 & 27 & 57.4 & 33 & 55.9 & 35 & 56.5 & 11 & 39.3 & 22 & 44.9 & 16 & 39.0 \\
\hline Head CT & 46 & 36.5 & 3 & 6.4 & 6 & 10.2 & 6 & 9.7 & 6 & 21.4 & 8 & 16.3 & 6 & 14.6 \\
\hline Head US & 39 & 31.0 & 12 & 25.5 & 15 & 25.4 & 16 & 25.8 & 7 & 25.0 & 14 & 28.6 & 12 & 29.3 \\
\hline Resident total & 126 & 61.5 & 47 & 75.8 & 59 & 68.6 & 62 & 67.4 & 28 & 29.5 & 49 & 41.9 & 41 & 36.6 \\
\hline Grand total & 205 & 100.0 & 62 & 100.0 & 86 & 100.0 & 92 & 100.0 & 95 & 100.0 & 117 & 100.0 & 112 & 100.0 \\
\hline
\end{tabular}

Note:-I-Phase indicates improvement phase; C-phase, control phase; RB-MRI, routine brain MRI.

${ }^{a}$ Duration of baseline was 3 months, followed by 3 months of improvement (each phase for 1 month), and 3 months of control phases (each phase for 1 month). 
Table 3: Number and percentage of each modality during different hours of the day from baseline to control phases ${ }^{\mathrm{a}}$

\begin{tabular}{|c|c|c|c|c|c|c|c|c|c|c|c|c|c|c|}
\hline & \multicolumn{2}{|c|}{ Baseline } & \multicolumn{2}{|c|}{ I-Phase I } & \multicolumn{2}{|c|}{ I-Phase II } & \multicolumn{2}{|c|}{ I-Phase III } & \multicolumn{2}{|c|}{ C-Phase I } & \multicolumn{2}{|c|}{ C-Phase II } & \multicolumn{2}{|c|}{ C-Phase III } \\
\hline & No. & $\%$ & No. & $\%$ & No. & $\%$ & No. & $\%$ & No. & $\%$ & No. & $\%$ & No. & $\%$ \\
\hline \multicolumn{15}{|l|}{ Work hours } \\
\hline RB-MRI & 27 & 19.7 & 6 & 14.3 & 6 & 9.4 & 6 & 9.8 & 12 & 16.4 & 6 & 8.6 & 11 & 12.5 \\
\hline UF-MRI & 34 & 24.8 & 21 & 50.0 & 40 & 62.5 & 26 & 42.6 & 34 & 46.6 & 38 & 54.3 & 44 & 50.0 \\
\hline Head CT & 28 & 20.4 & 2 & 4.8 & 1 & 1.6 & 1 & 1.6 & 8 & 11.0 & 1 & 1.4 & 6 & 6.8 \\
\hline Head US & 48 & 35.0 & 13 & 31.0 & 17 & 26.6 & 28 & 45.9 & 19 & 26.0 & 25 & 35.7 & 27 & 30.7 \\
\hline $\begin{array}{l}\text { Work hour total } \\
\text { After hours }\end{array}$ & 137 & 66.8 & 42 & 67.7 & 64 & 74.4 & \multicolumn{7}{|c|}{ After hours } & 78.6 \\
\hline RB-MRI & 7 & 20.6 & 2 & 14.3 & 3 & 25.0 & 2 & 13.3 & 3 & 23.1 & 3 & 13.6 & 4 & 25.0 \\
\hline UF-MRI & 2 & 5.9 & 8 & 57.1 & 5 & 41.7 & 9 & 60.0 & 6 & 46.2 & 9 & 40.9 & 8 & 50.0 \\
\hline Head CT & 19 & 55.9 & 2 & 14.3 & 3 & 25.0 & 3 & 20.0 & 1 & 7.7 & 6 & 27.3 & 2 & 12.5 \\
\hline Head US & 6 & 17.6 & 2 & 14.3 & 1 & 8.3 & 1 & 6.7 & 3 & 23.1 & 4 & 18.2 & 2 & 12.5 \\
\hline $\begin{array}{l}\text { After hours total } \\
\text { Weekend }\end{array}$ & 34 & 16.6 & 14 & 22.6 & 12 & 14.0 & 15 & 16.3 & 13 & 13.7 & 22 & 18.8 & 16 & 14.3 \\
\hline RB-MRI & 4 & 11.8 & 1 & 16.7 & 1 & 10.0 & & 0.0 & 3 & 33.3 & 2 & 8.0 & 1 & 12.5 \\
\hline UF-MRI & 1 & 2.9 & 2 & 33.3 & 4 & 40.0 & 10 & 62.5 & 2 & 22.2 & 9 & 36.0 & 2 & 25.0 \\
\hline Head CT & 15 & 44.1 & 1 & 16.7 & 2 & 20.0 & 3 & 18.8 & 1 & 11.1 & 7 & 28.0 & 1 & 12.5 \\
\hline Head US & 14 & 41.2 & 2 & 33.3 & 3 & 30.0 & 3 & 18.8 & 3 & 33.3 & 7 & 28.0 & 4 & 50.0 \\
\hline Weekend total & 34 & 16.6 & 6 & 9.7 & 10 & 11.6 & 16 & 17.4 & 9 & 9.5 & 25 & 21.4 & 8 & 7.1 \\
\hline Grand total & 205 & 100.0 & 62 & 100.0 & 86 & 100.0 & 92 & 100.0 & 95 & 100.0 & 117 & 100.0 & 112 & 100.0 \\
\hline
\end{tabular}

Note:- I-Phase indicates improvement phase; C-phase, control phase; RB-MRI, routine brain MRI.

a Duration of baseline was 3 months, followed by 3 months of improvement (each phase for 1 month), and 3 months of control phases (each phase for 1 month).

Table 4: Number and percentage of each modality from baseline to control phases ${ }^{\mathrm{a}}$

\begin{tabular}{|c|c|c|c|c|c|c|c|c|c|c|c|c|c|c|}
\hline & \multicolumn{2}{|c|}{ Baseline } & \multicolumn{2}{|c|}{ I-Phase I } & \multicolumn{2}{|c|}{ I-Phase II } & \multicolumn{2}{|c|}{ I-Phase III } & \multicolumn{2}{|c|}{ C-Phase I } & \multicolumn{2}{|c|}{ C-Phase II } & \multicolumn{2}{|c|}{ C-Phase III } \\
\hline & No. & $\%$ & No. & $\%$ & No. & $\%$ & No. & $\%$ & No. & $\%$ & No. & $\%$ & No. & $\%$ \\
\hline RB-MRI & 38 & 18.5 & 9 & 14.5 & 10 & 11.6 & 8 & 8.7 & 18 & 18.9 & 11 & 9.4 & 16 & 14.3 \\
\hline UF-MRI & 37 & 18.0 & 31 & 50.0 & 49 & 57.0 & 45 & 48.9 & 42 & 44.2 & 56 & 47.9 & 54 & 48.2 \\
\hline Head CT & 62 & 30.2 & 5 & 8.1 & 6 & 7.0 & 7 & 7.6 & 10 & 10.5 & 14 & 12.0 & 9 & 8.0 \\
\hline Head US & 68 & 33.2 & 17 & 27.4 & 21 & 24.4 & 32 & 34.8 & 25 & 26.3 & 36 & 30.8 & 33 & 29.5 \\
\hline
\end{tabular}

Note:-I-Phase indicates improvement phase; C-phase, control phase; RB-MRI, routine brain MRI.

${ }^{a}$ Duration of baseline was 3 months, followed by 3 months of improvement (each phase for 1 month), and 3 months of control phases (each phase for 1 month)

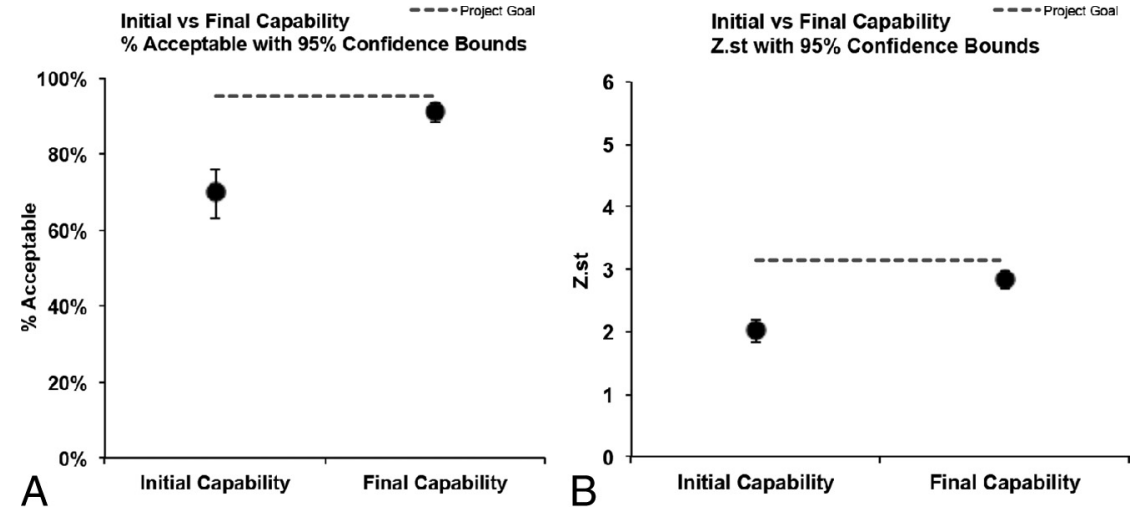

FIG 3. Attribute capability analysis demonstrates that our control data are almost at the $95 \%$ confidence bounds.

confidence bounds. Z St. analysis showed that the final control phase data almost reached 2 SDs, which was the $95 \%$ confidence bound, indicating a sustainable long-term stability of the process (Fig 4).

\section{DISCUSSION}

We reduced head CT orders by 75\% within 3 months from deploying the LSS project, surpassing our goal of reducing head CT orders by $50 \%$ in 6 months in children with hydrocephalus. More important, subsequent data collection/analysis during postintervention and control phases demonstrated that these changes were sustainable.
Head CT has been the mainstay of hydrocephalus imaging pathways to assess the ventricular size because it is easily accessible in most hospitals and provides quick information with a reasonably low total charge. Long-term survival has significantly improved in children with hydrocephalus with the use of modern CSF diversion techniques and advances in the care of shunts. Lowdose CT protocols have been used in some hospitals, including our own, following the principle of as low as reasonably achievable ${ }^{16}$; however, children with hydrocephalus will have multiple neuroimaging studies throughout their lives, increasing their total lifetime radiation exposure and consequently having a greater lifetime risk of radiation-induced brain cancers. $^{5-10}$ Therefore, elimination of radiation-based imaging techniques whenever possible is critical to ensure the long-term health of this population. Highlighting this belief, Koral et $\mathrm{al}^{7}$ recently found that there is an increased risk of developing fatal cancer if children with hydrocephalus undergo neuroimaging surveillance with head CTs. These and similar results have repeatedly been reported in the lay press, and as a result, both providers and patients' families are routinely demanding that radiation-free protocols be used whenever possible. 


\section{P Chart of Weekly Head CTs by Stage}

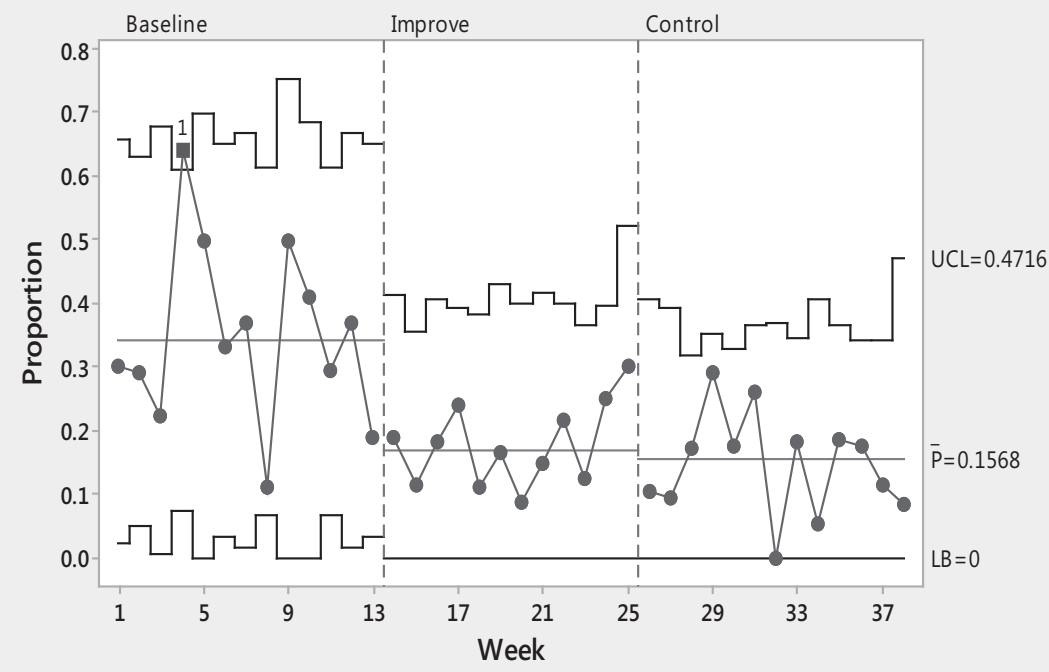

Tests performed with unequal sample sizes surgery resident as soon as the patients were transferred into the MR imaging scanner. The purchase of an additional shunt programmer for the PED enabled the pediatric neurosurgery staff to immediately check the shunt settings for patients on completion of imaging. We placed printed improved workflow charts at the ordering workstations of each unit to provide visual display.

Although there was some increase in head CTs and reduction in UF-MR imaging in the early control phase, the percentage of decrease in the head CTs did not fall below $50 \%$. The early control phase coincided with the new academic year, with influx of new residents and fellows, which, in part, may explain a temporary change. The electronic ordering system of our hospital was renewed during the course of this project. Therefore, before implementation of the new electronic ordering system, which coincided with the beginning of the control phase and the beginning of the new

Use of UF-MR imaging was first published in 2005 as a radiation-free alternative for neuroimaging in children with hydrocephalus and has been applied by the principal author since the late 1990s. ${ }^{11}$ Since then, UF-MR imaging has been validated with similar test characteristics for the evaluation of possible shunt failure (assessment of ventricular size and catheter visualization) compared with head CT. ${ }^{17-19}$ Diagnostic image quality and the short image-acquisition time of UF-MR imaging have also been reported. ${ }^{11-15}$ Despite the aforementioned publications, consistent nationwide use of this radiation-free neuroimaging technique has been somewhat limited. The palpable growing demand to improve quality and safety in imaging is now requiring radiologists to take leadership responsibilities, beyond simply reading a timely accurate radiology examination. ${ }^{20}$

Health care delivery systems are complex, and each institution has its unique resources, qualities, and challenges. In our study, we deployed an LSS methodology for solving our problem. In this article, we summarize and present our results in the framework of "how to do" this methodology. Lean methodology focuses on elimination of waste, which can be defective products, overproduction beyond demand, increased inventories, unnecessary motion/transport, and wait times as typically described in the manufacturing industry. In this study, the "defect" we sought to eliminate was the clinically unnecessary use of head CT. We reduced the overall radiation exposure to children significantly by using radiation-free imaging techniques. We completely eliminated the use of sedation/anesthesia by optimizing/shortening the UF-MR imaging protocol and educating technologists.

Unnecessary motion of the patient and transport staff was effectively eliminated by use of the emergency department MR imaging scanner and having our MR imaging technologists pick up and return the patients to their beds in the PED. We further reduced the patient's postimaging wait times by paging the neuro- academic year, the revision of the hydrocephalus imaging package with the pop-up information box was not accomplished. This likely explains the brief, transient relative increase in head CTs during this period. After implementation of the changes in the new electronic ordering system, the head CT and UF-MR imaging orders returned to a level similar to that seen during the early improvement phase. This difference highlights the importance/ value of the use of electronic health care systems for a successful execution of interventions that related to imaging appropriateness. Attribute capability analysis demonstrated that the control phase data almost reached the $95 \%$ confidence bounds.

Our study has several limitations. We identified our patients by using radiology information systems with the search term "hydrocephalus," which resulted in the inclusion of all patients with hydrocephalus regardless of whether they were shunted. We offered a timely service 24/7 to all providing units; however, we did not measure the total cycle time or throughput of the patients. Although we did not collect such data, the fact that the services have been used well in excess of our initial goal suggests that UF-MR imaging scans are being obtained in fashion timely enough for referring providers to effectively change their ordering patterns. We did not aim for $100 \%$ elimination of head CT, keeping in mind the potential need for head CT for medical reasons in select cases. We did not calculate the radiation savings for our patients, though this is a goal of our team for future.

\section{CONCLUSIONS}

We surpassed our goal of a 50\% reduction in head CT orders, achieving a sustained $75 \%$ reduction by effectively replacing most head CTs within a radiation- and sedation-/anesthesia-free UF-MR imaging protocol. Building a multidisciplinary team, having a tightly scoped project outline with clear goals aligned 
with our institutional and departmental priorities, and adhering closely to the DMAIC framework of LSS were all crucial in achieving our goal. Although factors may vary by hospital, similar LSS methodology could yield positive results for other indications such as adult hydrocephalus or other health care delivery systems.

\section{ACKNOWLEDGMENTS}

We acknowledge the contributions and support of each team member that we could not include in the authors list: Diane Novak (MR Imaging Manager), Bina Patel (Chief Pediatric MR Imaging Technologist and Manager), Beatrice Mudge (CT Manager), Robert DeJong (US Manager), Elyce Wolfgang (Pediatric Radiology Manager), and Allison Greene (medical student) from the Johns Hopkins Hospital; and Rich Hill from the Armstrong Institute for Patient Safety and Quality.

Disclosures: Eric M. Jackson—UNRELATED: Expert Testimony: I was an expert for a case of a neurosurgical patient with a diagnosis unrelated to this project. I have not been paid as of yet; Grants/Grants Pending: I had a grant from the institution for shunt development and have currently submitted requests for additional grants. I did not receive any money directly. ${ }^{*}$ Martin Bledsoe-UNRELATED: Expert Testimony: West Virginia University; ninety-minute case review. Daniel J. Durand-UNRELATED: Employment: Evolent Health, Comments: For 2 years I worked as a health care consultant, helping to improve the value delivered by imaging to patient populations. None of the pediatric MRI work described here overlaps any of my work at Evolent, however; Other: National Decision Support Company, Comments: unpaid advisor to a maker of clinical decision-support software designed to help ensure that the appropriate test is ordered for the right patient at the right time. They do not make a product for pediatric patients, and this work has no overlap with their Clinical Decision Support solution. George Jallo-UNRELATED: Expert Testimony: defense work for a law firm. Thierry A.G. Huisman—UNRELATED: Board Membership: Senior Editor for the American Journal of Neuroradiology. *Money paid to the institution.

\section{REFERENCES}

1. DelliFraine JL, Langabeer JR 2nd, Nembhard IM. Assessing the evidence of Six Sigma and Lean in the health care industry. Qual Manag Health Care 2010;19:211-25 CrossRef Medline

2. Vest JR, Gamm LD. A critical review of the research literature on Six Sigma, Lean and StuderGroup's Hardwiring Excellence in the United States: the need to demonstrate and communicate the effectiveness of transformation strategies in healthcare. Implement Sci 2009;4:35 CrossRef Medline

3. Simon TD, Riva-Cambrin J, Srivastava R, et al; Hydrocephalus Clinical Research Network. Hospital care for children with hydrocephalus in the United States: utilization, charges, comorbidities, and deaths. J Neurosurg Pediatr 2008;1:131-37 CrossRef Medline

4. Stein SC, Guo W. The prevalence of shunt-treated hydrocephalus: a mathematical model. Surg Neurol 2009;72:131-37 CrossRef Medline

5. Brenner D, Elliston C, Hall E, et al. Estimated risks of radiationinduced fatal cancer from pediatric CT. AJR Am J Roentgenol 2001; 176:289-96 CrossRef Medline

6. Brenner DJ, Hall EJ. Computed tomography: an increasing source of radiation exposure. N Engl J Med 2007;357:2277-84 CrossRef Medline

7. Koral K, Blackburn T, Bailey AA, et al. Strengthening the argument for rapid brain MR imaging: estimation of reduction in lifetime attributable risk of developing fatal cancer in children with shunted hydrocephalus by instituting a rapid brain MR imaging protocol in lieu of head CT. AJNR Am J Neuroradiol 2012;33:1851-54 CrossRef Medline

8. Durand DJ. A rational approach to the clinical use of cumulative effective dose estimates. AJR Am J Roentgenol 2011;197:160-62 CrossRef Medline

9. Pearce MS, Salotti JA, Little MP, et al. Radiation exposure from CT scans in childhood and subsequent risk of leukaemia and brain tumours: a retrospective cohort study. Lancet 2012;380:499-505 CrossRef Medline

10. Mathews JD, Forsythe AV, Brady Z, et al. Cancer risk in $\mathbf{6 8 0 , 0 0 0}$ people exposed to computed tomography scans in childhood or adolescence: data linkage study of 11 million Australians. $B M J$ 2013;346:f2360 CrossRef Medline

11. Ashley WW Jr, McKinstry RC, Leonard JR, et al. Use of rapid-sequence magnetic resonance imaging for evaluation of hydrocephalus in children. J Neurosurg 2005;103(2 suppl):124-30 Medline

12. Iskandar BJ, Sansone JM, Medow J, et al. The use of quick-brain magnetic resonance imaging in the evaluation of shunt-treated hydrocephalus. J Neurosurg 2004;101:147-51 Medline

13. Niederhauser BD, McDonald RJ, Eckel LJ, et al. Retrospective review of rapid pediatric brain MR imaging at an academic institution including practice trends and factors affecting scan times. AJNR Am J Neuroradiol 2013;34:1836-40 CrossRef Medline

14. O'Neill BR, Pruthi S, Bains H, et al. Rapid sequence magnetic resonance imaging in the assessment of children with hydrocephalus. World Neurosurg 2013;80:e307-312 CrossRef Medline

15. Rozovsky K, Ventureyra EC, Miller E. Fast-brain MRI in children is quick, without sedation, and radiation-free, but beware of limitations. J Clin Neurosci 2013;20:400-05 CrossRef Medline

16. Pindrik J, Huisman TA, Mahesh M, et al. Analysis of limited-sequence head computed tomography for children with shunted hydrocephalus: potential to reduce diagnostic radiation exposure. J Neurosurg Pediatr 2013;12:491-500 CrossRef Medline

17. Yue EL, Meckler GD, Fleischman RJ, et al. Test characteristics of quick brain MRI for shunt evaluation in children: an alternative modality to avoid radiation. J Neurosurg Pediatr 2015;15:420-26 CrossRef Medline

18. Mater A, Shroff M, Al-Farsi S, et al. Test characteristics of neuroimaging in the emergency department evaluation of children for cerebrospinal fluid shunt malfunction. CJEM 2008;10:131-35 Medline

19. Lehnert BE, Rahbar H, Relyea-Chew A, et al. Detection of ventricular shunt malfunction in the ED: relative utility of radiography, CT, and nuclear imaging. Emerg Radiol 2011;18:299-305 CrossRef Medline

20. Durand DJ, Kohli MD. Advanced practice quality improvement project: how to influence physician radiologic imaging ordering behavior. J Am Coll Radiol 2014;11:1155-59 CrossRef Medline 